\title{
Electron Crystallography
}

Electron Microscopy and Electron Diffraction

XIAODONG ZOU

SVEN HOVMÖLLER

PETER OLEYNIKOV 


\section{Confents}

1 Electron crystallography - an introduction 1

2 Fundamental crystallography 17

3 Crystal structure factors and symmetry 42

4 Fundamental transmission electron microscopy 72

5 Electron diffraction (ED) 90

6 Phase contrast, contrast transfer function (CTF) and high-resolution electron microscopy (HRTEM) 131

7 Solving crystal structures from HRTEM images by crystallographic image processing

8 Experimental procedures of crystallographic image processing

9 Solving crystal structures from ED data

10 Structure refinement

11 3D electron crystallography

12 Simulation of images and electron diffraction patterns

13 Applications of electron crystallography

Appendices

Index 